\title{
Design of Micro-ripple PWM-Cuk EBW High Voltage Stabilized Source
}

\author{
Yanping Qiao ${ }^{1, a}$, Jinhai Mo ${ }^{1, b}$ and Wei Li ${ }^{1, c}$ \\ ( College of Mechanical and Electrical Engineering, Guilin University of Electronic Technology, \\ Guilin 541004, Guangxi ) \\ a1196086709@qq.com, bgoldsea2005 @qq.com, 617912232@qq.com
}

Keywords: Ebw, Micro-ripple, Coupled inductor of circuit, Closed-loop control, Lag-lead

Abstract: The stability of the output voltage of the ebw's stable high voltage power is a prerequisite to ensure the quality of ebw. To solve this problem, an ebw high voltage stable power control system is designed. Main principle is that the new type of pwm-cuk converter structure is combined with coupled inductor, to reasonably allocate two inductors' coupling degree. At the same time, to develop the system's stability and control precision, the lag-lead control mode is utilized. Besides, main circuit and control circuit which are established and simulated by using saber and psim software. Theoretical analysis and simulations results show that the system not only possesses characteristic of micro ripple, but also has high control precision, good dynamic behavior, high energy conversion efficiency, low working condition requirements, high voltage stabilizing precision etc.

\section{Introduction}

Ebw high volgate stable power is mainly used for to provide acceleration voltage for electron gun, its performance directly determines the quality of ebw.

To improve the output stability, two conventional methods are adopted to reduce the ripple, one is to adopt technology of filter and compensation, the other is high frequency technology. Both of the two methods reduce the output ripple effectively. But from the perspective of the welding technical and requirement of ripple suppression, they don't obtain satisfactory results.Therefore, a kind of micro wave pwm-cuk ebw high voltage power control system is designed. A pwm-cuk transformation method is utilized in the main circuit; and coupled inductors are introduced to the cuk circuit. The circuit structure is simple, convenient debugging, easy to implement quick adjusting welding technology of electron beam, i.e. it meets the requirement for the stability of the ebw power.

\section{Ebw circuit principle analysis and modeling}

Main circuit is shown in fig. 1(a). Three-phase ac after isolation transformer booster and three-phase rectifier filter, high voltage pulsed dc is obtained. A cuk dc converter is designed; the duty ratio is controlled by the appropriate pwm signal, $\mathrm{U}_{\mathrm{o}}$ become about $60 \mathrm{kv}$, in energy storage capacitor $C_{0}$. In the fig. $1(a)$, the $R_{1}$ for over current protection, $R_{2}$ and $R_{3}$ for high voltage measurement, the resistance value of $R_{3}$ far less than $R_{2}$, benefited to measure voltage. $L_{x}$ is a choke inductor, to prevent short circuit. $\mathrm{V}_{\mathrm{x}}$ is a free-wheeling diode, to prevent producing high spike voltage. Ignoring auxiliary circuit and the secondary circuit element, get the equivalent circuit as shown in the fig. 1(b), the electron gun is equivalently converted to a variable resistance $\mathrm{R}$. 

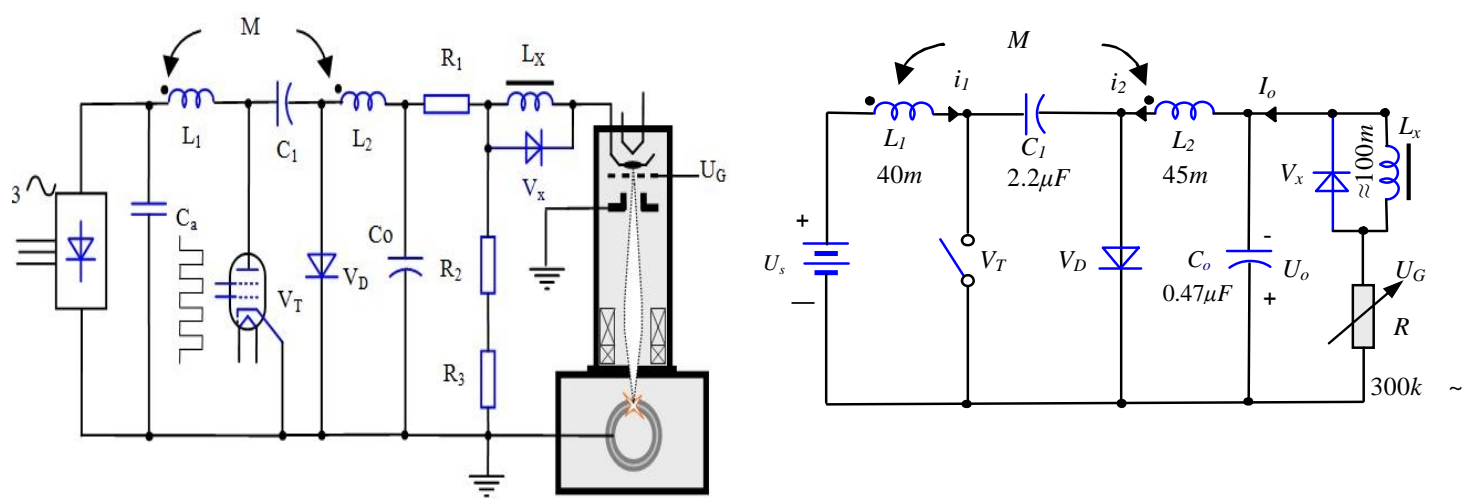

(a)

Fig.1 PWM - Cuk high voltage power

In the ebw power system, $\mathrm{I}_{\mathrm{o}}$ is less than $\mathrm{I}_{\mathrm{OK}}$. Consequently, the system stable state keeps current discontinuous, given as:

$$
I_{o}<I_{O K}=\frac{U_{o}}{2 L_{e} f}(1-d)^{2},
$$

Where $L_{e}$ represents equivalent inductor and $I_{O K}$ represents critical current, the value of $L_{e}$ is $\mathrm{L}_{1} \mathrm{~L}_{2} /\left(\mathrm{L}_{1}+\mathrm{L}_{2}\right)$.

New cuk circuit inductance $\mathrm{L}_{1}$ and $\mathrm{L}_{2}$ is coupling inductor, $\mathrm{M}$ is mutual inductance. Contrasted with the conventional cuk circuit, two independent inductors are changed to two coupling inductors coiled in an iron-core together. Theoretically, the ripple of input and output voltage can be reduced to infinitely small, by using two coupling inductors.

In the main circuit design, $\mathrm{L}_{1}$ is $40 \mathrm{mh}, \mathrm{L}_{2}$ is $45 \mathrm{mh}, \mathrm{M}$ is $35.56 \mathrm{mh}, \mathrm{K}$ is 0.83 . Comprehensive thinking about capacitor's voltage endurance and the manufacturing process and the requirement of low ripple, $C_{1}$ is $2.2 \mathrm{uf}, \mathrm{C}_{\mathrm{o}}$ is $0.47 \mathrm{uf}$. The basic parameters of ebw are given as, $\mathrm{U}_{\mathrm{s}}$ is $20 \mathrm{kv}, \mathrm{U}_{\mathrm{o}}$ is $60 \mathrm{kv}, \mathrm{d}$ is 0.73 , the rated power is $6 \mathrm{kw}$.

According to the theoretical extrapolation and results of calculation, simulation results are shown in fig. 2.

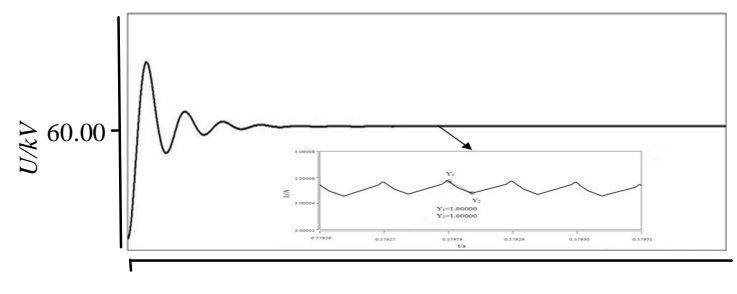

$t / s$

Fig.2 Cuk circuit output voltage waveform

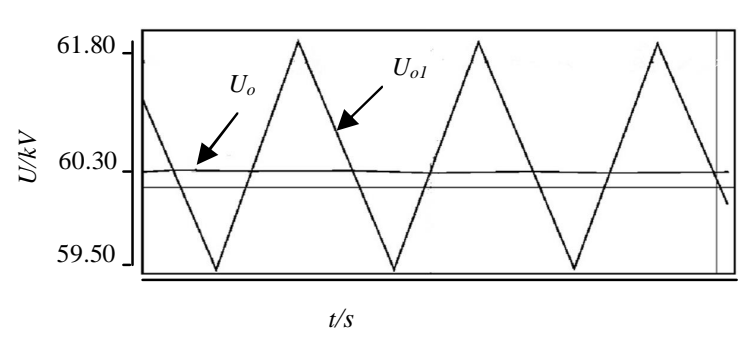

Fig.3 Cuk circuit output voltage ripple measurement comparison

Fig. 3 is cuk circuit output voltage ripple simulation comparison. In fig. $3, \mathrm{U}_{\mathrm{o} 1}$ is no coupled cuk circuit output voltage ripple, $\mathrm{U}_{\mathrm{o}}$ is coupled cuk circuit output voltage ripple value. The results of observing are given as, $\mathrm{U}_{\mathrm{o} 1}=2.3 \mathrm{kv}, \mathrm{U}_{\mathrm{o}}=0.3 \mathrm{kv}$. Contrasting with the results of fig. 3 , the coupled inductor can make cuk converter output reach micro ripple in theory.

When the $\mathrm{U}_{\mathrm{s}}-\mathrm{L}_{1}-\mathrm{V}_{\mathrm{T}}$ circuits and $\mathrm{R}-\mathrm{L}_{2}-\mathrm{C}_{1}-\mathrm{V}_{\mathrm{T}}$ circuits are pulled-in current simultaneously, the state equations of main circuit topology are given as: 


$$
\left\{\begin{array}{l}
L_{1} \frac{d i_{1}(t)}{d t}=u_{s}(t) \\
L_{2} \frac{d i_{2}(t)}{d t}=u_{c 1}(t)-i_{2}(t) R \\
C_{1} \frac{d u_{c 1}(t)}{d t}=-i_{2}(t)
\end{array}\right.
$$

From dT until $\mathrm{T}, \mathrm{V}_{\mathrm{T}}$ in the off state, $\mathrm{U}_{\mathrm{s}}-\mathrm{L}_{1}-\mathrm{C}_{1}-\mathrm{V}_{\mathrm{D}}$ circuit and $\mathrm{R}-\mathrm{L}_{2}-\mathrm{V}_{\mathrm{D}}$ circuit are respectively pulled-in current. The state equation of circuit topology is given as,

$$
\left\{\begin{array}{l}
L_{1} \frac{d i_{1}(t)}{d t}=u_{s}(t)-u_{c 1}(t) \\
L_{2} \frac{d i_{2}(t)}{d t}=-i_{2}(t) R \\
C_{1} \frac{d i_{2}(t)}{d t}=-i_{1}(t) R
\end{array},\right.
$$

Duty cycle is $d(t)$, set the $d(t)$ is $D, d$ 'is $1-D$. Disturbance is separated and then linearized, and the ac small signal matrix equations are obtained. Then from the control link to the output link, current of transfer function is obtained, as shown below:

$$
\left.\frac{\hat{i_{2}}(s)}{\hat{d}}\right|_{\hat{u}_{s}(s)=0}=\frac{L C U_{c 1} s^{2}-D L_{1}\left(I_{1}+I_{2}\right) s+\left(D D^{\prime}+D^{\prime 2}\right) U_{c 1}}{L_{1} L_{2} C_{1} s^{3}+L_{1} C_{1} R_{S^{2}}+\left(D^{2} L_{1}+D^{\prime 2} L_{2}\right) s+R D^{\prime}},
$$

\section{The working principle of voltage stability control system}

Pwm method is used in this design. After the signals of feedback and given pass though lag -lead compensation correction link, they are modulated to two complementary pwm pulse by t1494. Besides, excessive surge current affects the normal work of the circuit system. To limit it, current-limiting devices are needed to be designed.

T1494 is chosen as the pwm pulse width modulator. Amplifier of t1494 connected with the hall current sensor realized the function of over-current protection. Its non-contact measurement can ensure the reliable isolation of control circuit and main circuit, and realize fast dynamic response.

The currents are limited accurately, and can effectively prevent circuit from inducing short-circuit, i.e. over-current protection is easy to implement. In the actual circuit system, because of the existence of the circuit itself dynamic factors and the external disturbance (such as the duty ratio $d$ mutations, three-phase power voltage fluctuation and rectifier circuit voltage fluctuation, etc), a closed-loop system is needed to suppress the interference and make the output voltage stability. Based on the established cuk circuit transfer function expression of small signal mathematical model, the key circuit parameters into the function, psim software is used to simulate and analysis. Bode diagrams of system open loop transfer function from the simulation results are shown in fig. 4(a). 


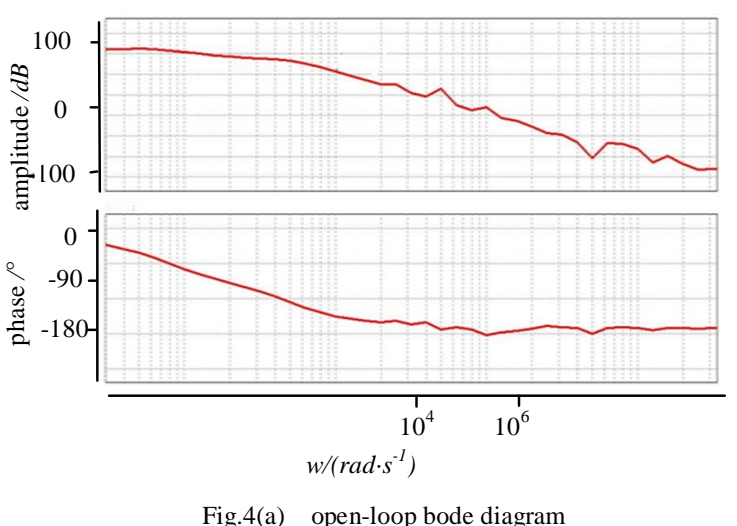

Fig.4(a) open-loop bode diagram



Fig.4(b) closed-loop bode diagram

The above fig. 4(a) illustrates the phase margin of open-loop bode diagram is $-5^{\circ}$. Reversely, without compensation, the system output voltage is unstable. According to the simulation test, the system controlling is incorrect by solely using lag compensation or lead compensation. Therefore, the lag-lead correction compensation mode is used for the system. Adjusted the new control system is shown in fig.5.

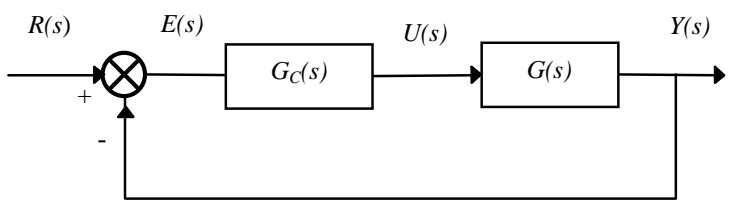

Fig.5 Control system

In fig. 5 control system, $\mathrm{G}$ (s) is the multiply of the transfer functions of the three links. The three links are the pulse width modulation link, the test feedback link and the cuk main circuit link. Pulse width modulation transfer function is $\mathrm{Gm}(\mathrm{s}), \mathrm{Gm}(\mathrm{s})$ is $1 / \mathrm{Vm}, \mathrm{Vm}$ is peak-to-peak value of the sawtooth wave. $\mathrm{H}(\mathrm{s})$ is to detect feedback loop transfer function, $\mathrm{H}(\mathrm{s})=5 / 60000=1 / 12000$. Gc (s) is the transfer function of lag - lead compensation correction link, the phase margin and amplitude margin and steady state error (gain) etc are used for its performance index.

The principle of lag - lead compensation is that the lag part increases the phase margin of the system and its lead part improves the stability performance of the system. The system steady-state error is ess, open loop gain is $\mathrm{k}$.

$$
e_{s s} \leq 0.01, e_{s s}=1 /(1+K),
$$

Where $\mathrm{k}$ is 99 , for the convenience of calculation, take the $\mathrm{k}$ value is 100 . After debugging and modification, the transfer function of the lag - lead compensation link is shown below:

$$
G_{C}(s)=\frac{1+500 s}{1+5000 s} \cdot \frac{1+2 \times 10^{-4} s}{1+2 \times 10^{-5} s},
$$

After introducing lag-lead compensation correction link, bode diagram of the closed-loop system is shown in fig.4(b). The phase margin is $42^{\circ}$ and amplitude margin is $27.1 \mathrm{db}$. Thus, utilizing the lag - lead compensation link, the system is stable. The rationality of the lag - lead compensation is verified by inputting step signal suddenly. The output voltage of this system is given as the fig.6. Dynamic response of transition time is short. And system overshoot is small. 


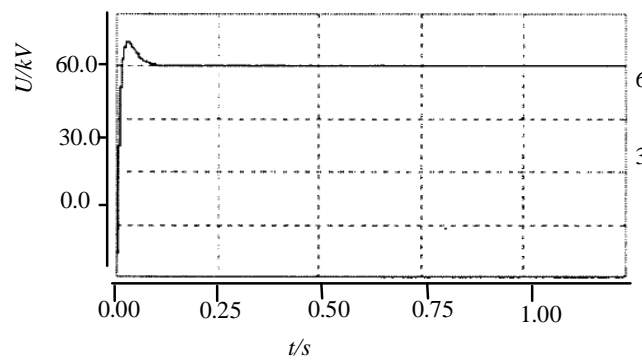

Fig.6 step disturbance



Fig. $7 \stackrel{t / s}{\text { input disturbance }}$

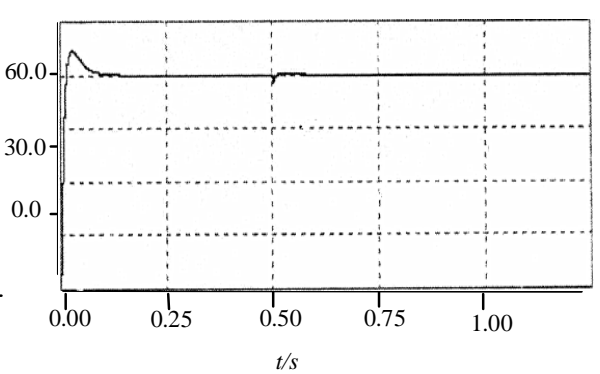

Fig.8 load disturbances

To verify this system's disturbance resistance characteristic, $\pm 300 \mathrm{~V}$ disturbance as the system' input voltage disturbance. The simulation results are shown in fig. 7. The load is suddenly connected to the original system in parallel. Therefore, the load is increased suddenly. The simulation results are as shown in fig.8. According to fig. 7 and 8 , although the system is influenced by the positive and negative voltage and load disturbances, system can reach steady state through the automatic adjustment, and the output voltage has not changed. As such, the parameters of modulator are reasonable, i.e. the system has good stability.

\section{Conclusions}

(1) Coupling inductance is applied to the pwm-cuk circuit of ebw power realized the request of micro wave output. Besides, small signal mathematical model of Cuk circuit is established. Furthermore, this system is simple and works well.

(2) Lag - lead correction is utilized in the feedback compensation link of the system. The reasonability of correction parameters and the selected methods are verified by system closed-loop control bode diagrams and the disturbance waveform figures. Moreover, the micro wave output characteristic of this system is also verified by the experimental results.

\section{Acknowledgements}

This work was financially supported by Guangxi natural science foundation of China (2013 gxnsfaa019301), guilin university of electronic technology post-graduate innovation projects (GDYCSZ 201446).

\section{References}

[1] Shouqi Wei: Electric Welding Machine, Vol. 34 (2004), p. 56 "in chinese"

[2] Peishan Dai: Electric Welding Machine, Vol. 2 (1998), p. 36 "in chinese"

[3] Hanmin Ye: High Voltage Engineering, Vol. 6 (1999), p. 50 "in chinese”

[4] J Chen: Power Electronics, Vol. 3 (2002), p. 1 "in chinese"

[5] Hanmin Ye: Electricity Technology Magazine, Vol. 10 (2000), p. 1 "in chinese"

[6] Jai P. Agrawal: Power Electronic System-Theory and Design. Vol. 30 (1990), p. 5

[7] W.H. Mangione-Smith: IEEE Computer, Vol. 30 (1997), p. 12 